\title{
Dietary and Lifestyle Factors Associated with Gastric and Pancreatic Cancers: A Case-Control Study
}

\author{
Narmeen Al-Awwad', Sabika Allehdan', Tareq Al-Jaberi ${ }^{2}$, Ahmad Hushki $^{3}$, Yazan Albtoush ${ }^{4}$, \\ Kamal Bani-Hani ${ }^{5}$, and Reema Fayez Tayyem ${ }^{6}$ \\ ${ }^{1}$ Department of Clinical Nutrition and Dietetics, Faculty of Applied Health Sciences and \\ ${ }^{5}$ Department of General and Special Surgery, Faculty of Medicine, The Hashemite University, Zarqa 13133, Jordan \\ ${ }^{2}$ Department of Surgery and Urology, Faculty of Medicine, Jordan University of Science and Technology, Irbid 22110, Jordan \\ ${ }^{3}$ Gastroenterology Division, King Hussein Cancer Center, Amman 11941, Jordan \\ ${ }^{4}$ Gastroenterology Division, Al-Bashir Hospital, Amman 11152, Jordan \\ ${ }^{6}$ Department of Human Nutrition, College of Health Science, Qatar University, Doha 2713, Qatar
}

\begin{abstract}
Gastric cancer (GC) and pancreatic cancer (PC) are the third and seventh most likely cancers to cause death worldwide. We aimed to determine the dietary and lifestyle factors of patients with GC or PC and their associated risk among Jordanians. This case-control study enrolled 587 adults (patients with PC, 101; patients with GC, 172; healthy controls, 314) between March 2015 and August 2018, who were assessed using interview-based personal and physical activity questionnaires. Multivariable logistic regression models were taken as measures for predictors of GC and PC risk. We showed that GC and PC patients had higher pre-diagnosis body-mass indexes, a greater proportion smoked and had a family history of cancer than controls. Furthermore, consumption of two snacks [odds ratios $(\mathrm{OR})=0.44,95 \%$ confidence intervals $(\mathrm{CI}): 0.23 \sim 0.85]$, three snacks $(\mathrm{OR}=0.04,95 \% \mathrm{CI}: 0.01 \sim 0.23)$ and no meals eaten outside $(\mathrm{OR}=0.31,95 \% \mathrm{CI}$ : $0.09 \sim 0.99)$ showed a protective effect against GC, and consumption of three snacks ( $\mathrm{OR}=0.08,95 \% \mathrm{CI}$ : $0.02 \sim 0.40)$ reduced significantly the risk of PC. These results suggest that bodyweight, physical activity, smoking, and family history of cancer are among factors that affect GC and PC risk among Jordanians.
\end{abstract}

Keywords: body mass index, gastric cancer, pancreatic cancer, physical activity, smoking

\section{INTRODUCTION}

Gastric cancer (GC) is the third most common type of cancer to cause death and the fifth most common type worldwide (Ferlay et al., 2015). From 2007 to 2016, the incidence rate for pancreatic cancer (PC) increased by $0.7 \%$ per year in white individuals and $0.3 \%$ per year in black individuals (American Cancer Society, 2020). However, from 2008 to 2017, mortality trends demonstrated a slight increase in death rate for PC (by $0.4 \%$ per year) in white individuals and a slight decrease (by $0.5 \%$ per year) in black individuals (American Cancer Society, 2020).

GC (Carcas, 2014) and PC (Costello, 2018) are heterogeneous diseases that are commonly diagnosed in their late stages (Lee and Derakhshan, 2013). Therefore, careful attention should be given to prevent GC and PC development since prevention and early diagnosis are vital approaches for controlling and reducing mortality rates (American Cancer Society, 2020).

Established risk factors for gastric and pancreatic cancers include smoking, excess body weight, high salt intake, alcohol, physical activity, and infection by Helicobacter pylori (American Cancer Society, 2020). However, studies show contrasting results, some documenting that smoking decreases survival (Zhao et al., 2020) and others showing no significant association (Trivers et al., 2005; Sundelöf et al., 2008; Ferronha et al., 2012). An estimated $5 \%$ of cancers in men and $11 \%$ in women are related to excess body weight (Islami et al., 2018). Excess body weight (i.e., being overweight or obese) is associated with an increased risk of many types of cancers, including gastric and pancreatic cancers (American Cancer Society, 2020).

An estimated 3\% of cancer cases are attributed to phys- 
ical inactivity (Islami et al., 2018). Physical activity is associated with a reduced risk of stomach and pancreatic cancers (World Cancer Research Fund/American Institute for Cancer Research, 2018). A greater amount of sedentary behaviour may increase risk of several types of cancers, including GC and PC (2018 Physical Activity Guidelines Advisory Committee, 2018). Furthermore, cancer patients who are physically active are less likely to have adverse effects and die from cancers than those who are inactive (Cormie et al., 2017). In Jordan, no previous studies have evaluated the associations between GC or PC and possible modifiable and non-modifiable risk factors. The present study was conducted to investigate the potential association between these lifestyle factors and dietary habits and the risk of GC or PC among Jordanians.

\section{MATERIALS AND METHODS}

\section{Study design and participants}

This case-control study was conducted in Jordan between March 2015 and August 2018. In total, 114 patients with medically confirmed PC and 186 patients with medically confirmed GC were invited to participate, and 101 patients with PC and 172 patients with GC enrolled. The control group consisted of 314 individuals without $\mathrm{PC}$ and GC, who were conveniently selected from the community. Population-based controls were frequency matched to cases based on age, gender, occupation, and marital status.

Inclusion criteria included Jordanian nationality, aged 18 years or older at enrolment, able to communicate verbally, and absence of any chronic diseases that require dietary modifications, such as kidney disease, liver disease, and celiac disease. For the GC and PC cases, patients must have been diagnosed within the last six months. Exclusion criteria included individuals who were critically ill, hospitalized, or unable to communicate verbally. Informed consent was obtained from all participants before enrolment.

Cases were enrolled from four hospitals: King Hussein Cancer Center, King Abdullah University Hospital, Jordan University Hospital, and Al-Bashir Hospital. The study protocol conformed to the ethical guidelines of the 1975 Declaration of Helsinki and was approved by the Ethics Committee of the King Hussein Cancer Center (IRB No. 15 KHCC 03, Amman, Jordan).

\section{Data collection}

Data were obtained by completing personal and physical activity questionnaires, which were completed during face-to-face interviews and filed by trained research assistants.
The personal questionnaire included questions related to age, gender, marital status, educational attainment, employment status, family income/month, smoking status, previous and current health problems, family history of cancer, and the presence or absence of stomach pain and ulcers. Smoking status included non-smoker, current smoker, previous smoker, and passive smoker (secondhand smoker) (Thornton et al., 1994). Information about dietary habits, such as consumption of main meals and snacks, frequency of skipping meals (breakfast, lunch, or dinner), frequency of eating problems, and daily water intake were also collected.

\section{Anthropometric measurements}

Participants' current body weight and height were measured using standardized techniques and calibrated tools by trained research assistants. Body weight was measured to the nearest $0.1 \mathrm{~kg}$ with minimal clothing and without shoes using a calibrated scale (seca GmbH \& Co. KG, Hamburg, Germany). Height was measured to the nearest $\mathrm{cm}$ when participants in the full standing position without shoes using a calibrated measuring rod (seca $\mathrm{GmbH}$ \& Co. KG). Body mass index (BMI) was computed as the ratio of weight $(\mathrm{kg})$ to height squared $(\mathrm{m})$ (Lee and Nieman, 2013), and classified according to World Health Organization guidelines (WHO, 2002). However, the usual body weight at pre-diagnosis was self-reported from the cases and controls.

\section{Physical activity questionnaire}

A validated 7-day Physical Activity Recall (PAR) questionnaire was used to estimate participant's physical activity levels. The 7-day PAR is a structured questionnaire that depends on participant's recall of time spent on physical activity over a 7-day period (Sallis et al., 1985). It assesses physical activity intensity (e.g. from aerobic exercise, work-related activities, walking, gardening, recreation, and leisure activities). The frequency, intensity, duration, and type of physical activity are typically taken into consideration to estimate the level of physical activity. The number of hours spent sleeping and undertaking different levels of activity levels were assessed and converted into metabolic equivalents (MET). According to the scoring instructions, sleeping was assigned a value of 1.0 MET, light activity a value of 1.5 METs, moderate activity a value of $4.0 \mathrm{METs}$, and very hard activity a value of 7.0 METs or greater (Thompson et al., 1982). Physical activity was calculated as the time (min) dedicated to each activity multiplied by the calculated MET and multiplied by the number of days the activity was undertaken during the week, as follows: MET level $\times$ min of activity/day $\times$ days per week (Sallis et al., 1985). Physical activity level was expressed as continuous data in MET-min/week, and as categorical scores [inactive, min- 
imally active, and Health-Enhancing Physical Activity (HEPA) active] based on the standard scoring protocol of International Physical Activity questionnaire. Participants were considered inactive if their activity levels were too low to meet creteria of minimally active or HEPA active. The minimally active category included participants who met any of the following conditions: (1) vigorous activity on 3 or more days for at least 20 min per day; (2) moderate intensity activity or walking on 5 or more days for at least 30 min per day, or (3) vigorous intensity activities on 5 or more days, achieving a minimum of 600 MET-min/week. The HEPA active catogory included participants who performed either vigorous intensity activity on at least 3 days and accumulated at least 1,500 MET$\mathrm{min} /$ week, or participants who performed a combination of walking, moderate intensity or vigorous intensity activities on at least 7 days, achieving a minimum of at least 3,000 MET-min/week (Forde, 2018).

\section{Statistical analysis}

Statistical analysis was performed using the Statistical Package for the Social Sciences (SPSS) version 27 (IBM Corporation, Armonk, NY, USA). Frequencies and percentages were calculated to describe categorical variables. Pearson chi-square tests was used to detect differences in personal characteristics, lifestyle variables, and eating habits among study participants. Means and standard error of the mean (SEM) were calculated for the continuous variables. One-way analysis of variance (ANOVA) and Fisher's least significant difference post hoc tests were used to calculate differences in continuous variables between gastric and pancreatic cases and controls. The significance level was set at $P<0.05$. Odds ratios (OR) and $95 \%$ confidence intervals ( $\mathrm{CI}$ ) from multivariable logistic regression models were taken as measures for predictors of gastric and pancreatic cancers risk.

\section{RESULTS}

Table 1 shows the characteristics of participants with GC $(n=172)$ and PC $(n=101)$, and controls $(n=314)$. Participants with GC and PC had a similar age, height, and income as controls. The pre-diagnosis BMI for participants with both GC and PC were significantly higher than controls. However, current body weight and BMI for both types of cancer were significantly lower than for controls.

The number of cigarettes smoked per day was $13.5 \pm 1.6$ for participants with GC, $10.8 \pm 1.6$ for participants with PC, and 9.0 \pm 0.9 for controls. The number of cigarette smokers in the GC group was significantly higher than for controls. The duration of smoking was $12.4 \pm 17.1$ and $13.1 \pm 26.7$ years for GC and PC cases, respectively, and was significantly higher than for controls $(8.8 \pm 14.7$ years). Total physical activity METs (MET-min/week) were $1,031.5 \pm 42.7,952.9 \pm 47.2$, and $1,314.7 \pm 45.6$ for GC and PC cases, and controls, respectively. However, all participants were considered minimally active (Table 1).

Table 2 shows the demographic and lifestyle factors of participants with GC and PC, and controls. Most GC cases (57.1\%) and PC cases $(52.6 \%)$ were considered normal according to their BMIs. However, most were previously considered overweight (GC, 40.4\%; PC, $41.1 \%$ ) or obese (GC, 41.0\%; PC, 41.1\%). The Majority of GC (67.9\%) and PC (73.9\%) cases were considered stage 4 .

The total number of participants who smoked (including current, previous, and passive smokers) was significantly higher for GC (52.0\%) and PC (46.5\%) cases than controls (39.5\%). However, no significant difference was detected in the daily number of cigarettes smoked by the subjects amongst study groups.

A greater number of participants with GC $(48.6 \%)$ and PC (48.5\%) had a family history of cancer compared with the control group (31.8\%). Participants with GC reported

Table 1. Characteristics of the study participants

\begin{tabular}{lcccc}
\hline & $\begin{array}{c}\text { Gastric cancer cases } \\
(\mathrm{n}=172)\end{array}$ & $\begin{array}{c}\text { Pancreatic cancer } \\
\text { cases }(\mathrm{n}=101)\end{array}$ & Controls (n=314) & $P$-value \\
\hline Age (years) & $54.1 \pm 1.0$ & $56.97 \pm 1.2$ & $54.0 \pm 0.7$ & 0.093 \\
Height (cm) & $167.9 \pm 0.7$ & $166.2 \pm 0.9$ & $168.0 \pm 0.5$ & 0.228 \\
Pre-diagnosis body weight (kg) & $85.3 \pm 1.6^{\mathrm{a}}$ & $83.4 \pm 2.0^{\mathrm{ab}}$ & $79.4 \pm 1.2^{\mathrm{b}}$ & 0.009 \\
Current weight $(\mathrm{kg})$ & $70.6 \pm 1.3^{\mathrm{b}}$ & $69.4 \pm 1.4^{\mathrm{b}}$ & $80.9 \pm 0.9^{\mathrm{a}}$ & 0.001 \\
Pre-diagnosis BMI (kg/m $\left.\mathrm{m}^{2}\right)$ & $30.1 \pm 0.5^{\mathrm{a}}$ & $30.2 \pm 0.7^{\mathrm{a}}$ & $28.3 \pm 0.4^{\mathrm{b}}$ & 0.008 \\
Current BMI (kg/m $\left.{ }^{2}\right)$ & $25.0 \pm 0.5^{\mathrm{b}}$ & $25.1 \pm 0.5^{\mathrm{b}}$ & $28.7 \pm 0.3^{\mathrm{a}}$ & 0.001 \\
Income (JD) & $674.0 \pm 79.6$ & $700.1 \pm 74.7$ & $575.9 \pm 36.0$ & 0.266 \\
Number of cigarettes/d & $13.5 \pm 1.6^{\mathrm{a}}$ & $10.8 \pm 1.6^{\mathrm{ab}}$ & $9.0 \pm 0.9^{\mathrm{b}}$ & 0.028 \\
Duration of smoking (years) & $12.4 \pm 17.1^{\mathrm{a}}$ & $13.1 \pm 26.7^{\mathrm{a}}$ & $8.8 \pm 14.7^{\mathrm{b}}$ & 0.036 \\
Total physical activity (MET-min/week) & $1,031.5 \pm 42.7^{\mathrm{b}}$ & $952.9 \pm 47.2^{\mathrm{b}}$ & $1,314.7 \pm 45.6^{\mathrm{a}}$ & 0.001 \\
\hline
\end{tabular}

${ }^{1)}$ Inactive: not fitting in "minimally active [at least 600 metabolic equivalents (MET)-min/week]" or "HEPA active (more than 3,000 MET-min/week)".

Each value is represented as mean \pm SEM.

Means with different letters $(a, b)$ within the same row are significantly different $(P<0.05)$.

BMI, body mass index; JD, Jordan dinar. 
Table 2. Demographic and lifestyle factors of patients with gastric cancer and pancreatic cancer

\begin{tabular}{|c|c|c|c|c|}
\hline Demographic and lifestyle factors & $\begin{array}{c}\text { Gastric cancer } \\
(n=173, \%)\end{array}$ & $\begin{array}{c}\text { Pancreatic cancer } \\
(n=101, \%)\end{array}$ & $\begin{array}{c}\text { Control } \\
(n=314, \%)\end{array}$ & $P$-value \\
\hline Gender & & & & 0.853 \\
\hline Male & 107 (61.8\%) & 59 (58.4\%) & 191 (60.8\%) & \\
\hline Female & $66(38.2 \%)$ & $42(41.6 \%)$ & $123(39.2 \%)$ & \\
\hline Pre-diagnosis BMI categories & & & & 0.163 \\
\hline$<18.5$ & $0(0.0 \%)$ & $1(1.1 \%)$ & $6(2.1 \%)$ & \\
\hline $18.5 \sim 24.9$ & $29(18.6 \%)$ & $16(16.8 \%)$ & $73(25.9 \%)$ & \\
\hline $25.0 \sim 29.9$ & $63(40.4 \%)$ & $39(41.1 \%)$ & 97 (34.4\%) & \\
\hline$>30.0$ & $64(41.0 \%)$ & 39 (41.1\%) & $106(37.6 \%)$ & \\
\hline Missing ${ }^{1)}$ & 17 & 6 & 32 & \\
\hline Current BMI categories & & & & 0.001 \\
\hline$<18.5$ & $9(5.8 \%)$ & $6(6.2 \%)$ & $0(0.0 \%)$ & \\
\hline $18.5 \sim 24.9$ & $89(57.1 \%)$ & $51(52.6 \%)$ & $85(27.8 \%)$ & \\
\hline $25.0 \sim 29.9$ & $30(19.2 \%)$ & $21(21.6 \%)$ & $108(35.3 \%)$ & \\
\hline$>30.0$ & $28(17.9 \%)$ & $19(19.6 \%)$ & $113(36.9 \%)$ & \\
\hline Missing & 17 & 4 & 8 & \\
\hline Cancer stages & & & & 0.001 \\
\hline No cancer & $0(0.0 \%)$ & $0(0.0 \%)$ & $314(100.0 \%)$ & \\
\hline Stage 1 & $0(0.0 \%)$ & $1(1.1 \%)$ & 0 (0.0\%) & \\
\hline Stage 2 & $2(1.4 \%)$ & $1(1.1 \%)$ & $0(0.0 \%)$ & \\
\hline Stage 3 & $43(30.7 \%)$ & $21(23.9 \%)$ & $0(0.0 \%)$ & \\
\hline Stage 4 & $95(67.9 \%)$ & $65(73.9 \%)$ & $0(0.0 \%)$ & \\
\hline Missing & 33 & 13 & 0 & \\
\hline Marital status & & & & 0.714 \\
\hline Married & $148(85.5 \%)$ & 87 (86.1\%) & $273(86.9 \%)$ & \\
\hline Single & $8(4.6 \%)$ & $5(5.0 \%)$ & 20 (6.4\%) & \\
\hline Divorced & $3(1.7 \%)$ & $3(3.0 \%)$ & $7(2.2 \%)$ & \\
\hline Widowed & $14(8.1 \%)$ & $6(5.9 \%)$ & $14(4.5 \%)$ & \\
\hline Education level & & & & 0.348 \\
\hline Illiterate & $10(5.8 \%)$ & $7(6.9 \%)$ & $18(5.8 \%)$ & \\
\hline Less than high school & 54 (31.4\%) & $24(23.8 \%)$ & $80(25.6 \%)$ & \\
\hline High school & $43(25.0 \%)$ & $14(13.9 \%)$ & 72 (23.0\%) & \\
\hline Diploma & 25 (14.5\%) & $18(17.8 \%)$ & $56(17.9 \%)$ & \\
\hline Bachelor & 34 (19.8\%) & $30(29.7 \%)$ & 71 (22.7\%) & \\
\hline Master's degree & $4(2.3 \%)$ & $5(5.0 \%)$ & $13(4.2 \%)$ & \\
\hline Doctorate degree & $2(1.2 \%)$ & $3(3.0 \%)$ & $3(1.0 \%)$ & \\
\hline Missing & 1 & 0 & 1 & \\
\hline Employment status & & & & 0.778 \\
\hline Yes & $82(47.7 \%)$ & 45 (45.0\%) & 153 (49.0\%) & \\
\hline No & $90(52.3 \%)$ & $55(55.0 \%)$ & 159 (51.0\%) & \\
\hline Missing & 1 & 1 & 2 & \\
\hline Smoking status & & & & 0.001 \\
\hline Total number of the smokers & 90 (52.0\%) & $47(46.5 \%)$ & $124(39.5 \%)$ & \\
\hline Current smoker & $56(32.4 \%)$ & $38(37.6 \%)$ & 99 (31.5\%) & \\
\hline Previous smoker & $23(13.3 \%)$ & $3(3.0 \%)$ & $13(4.1 \%)$ & \\
\hline Passive smoker & $11(6.3 \%)$ & $6(5.9 \%)$ & $12(3.8 \%)$ & \\
\hline Non-smoker & $83(48.0 \%)$ & $54(53.5 \%)$ & $190(60.5 \%)$ & \\
\hline Number of cigarettes/d & & & & 0.467 \\
\hline$<1$ & 95 (56.2\%) & $60(60.0 \%)$ & $206(65.6 \%)$ & \\
\hline $2 \sim 5$ & $6(3.6 \%)$ & $3(3.0 \%)$ & $12(3.8 \%)$ & \\
\hline $6 \sim 10$ & $5(3.0 \%)$ & $3(3.0 \%)$ & $12(3.8 \%)$ & \\
\hline $11 \sim 20$ & $23(13.6 \%)$ & $17(17.0 \%)$ & $42(13.4 \%)$ & \\
\hline $21 \sim 40$ & $28(16.6 \%)$ & $12(12.0 \%)$ & $29(9.2 \%)$ & \\
\hline$>41$ & $12(7.1 \%)$ & $5(5.0 \%)$ & 13 (4.1\%) & \\
\hline Missing & 4 & 1 & 0 & \\
\hline
\end{tabular}


Table 2. Continued

\begin{tabular}{|c|c|c|c|c|}
\hline Demographic and lifestyle factors & $\begin{array}{c}\text { Gastric cancer } \\
(n=173, \%)\end{array}$ & $\begin{array}{c}\text { Pancreatic cancer } \\
(n=101, \%)\end{array}$ & $\begin{array}{c}\text { Control } \\
(n=314, \%)\end{array}$ & $P$-value \\
\hline Hookah & & & & 0.428 \\
\hline Smoker & $7(4.0 \%)$ & $6(5.9 \%)$ & $27(8.6 \%)$ & \\
\hline Non-smoker & $163(94.2 \%)$ & 93 (92.1\%) & 282 (89.8\%) & \\
\hline Previous smoker & $3(1.7 \%)$ & $2(2.0 \%)$ & $5(1.6 \%)$ & \\
\hline Hookah smoking/d & & & & 0.381 \\
\hline 0 & $163(97.6 \%)$ & 93 (93.9\%) & 284 (93.7\%) & \\
\hline 1 & $3(1.8 \%)$ & $5(5.1 \%)$ & $13(4.3 \%)$ & \\
\hline$\geq 2$ & $1(0.6 \%)$ & $1(1.0 \%)$ & $6(2.0 \%)$ & \\
\hline Missing & 6 & 2 & 11 & \\
\hline Health problem & & & & 0.195 \\
\hline No & 95 (54.9\%) & $45(44.6 \%)$ & $151(48.1 \%)$ & \\
\hline Yes & $78(45.1 \%)$ & $56(55.4 \%)$ & 163 (51.9\%) & \\
\hline Type of health problem & & & & 0.004 \\
\hline No & 95 (54.9\%) & $45(44.6 \%)$ & 151 (48.1\%) & \\
\hline Diabetes mellitus & $33(19.1 \%)$ & $36(35.6 \%)$ & 81 (25.8\%) & \\
\hline Heart & $10(5.8 \%)$ & $10(9.9 \%)$ & $19(6.1 \%)$ & \\
\hline Hypertension & $30(17.3 \%)$ & 9 (8.9\%) & $40(12.7 \%)$ & \\
\hline Other & $5(2.9 \%)$ & $1(1.0 \%)$ & $23(7.3 \%)$ & \\
\hline Family history of cancer & & & & 0.001 \\
\hline Yes & $84(48.6 \%)$ & $49(48.5 \%)$ & 100 (31.8\%) & \\
\hline No & 89 (51.4\%) & $52(51.5 \%)$ & $214(68.2 \%)$ & \\
\hline Cancer type for patient's family & & & & 0.001 \\
\hline Absent & 89 (51.4\%) & 52 (51.5\%) & $214(68.2 \%)$ & \\
\hline Gastric & 7 (9.8\%) & $4(4.0 \%)$ & $10(3.2 \%)$ & \\
\hline Colon and/or rectal & $9(5.2 \%)$ & $1(1.0 \%)$ & $13(4.1 \%)$ & \\
\hline Pancreatic & $1(0.6 \%)$ & $3(3.0 \%)$ & $1(0.3 \%)$ & \\
\hline Bone cancer & $4(2.3 \%)$ & $3(3.0 \%)$ & $6(1.9 \%)$ & \\
\hline Lung & $5(2.9 \%)$ & $6(5.9 \%)$ & $11(3.5 \%)$ & \\
\hline Leukaemia & $3(1.7 \%)$ & $1(1.0 \%)$ & $10(3.2 \%)$ & \\
\hline Breast & $16(9.2 \%)$ & $7(6.9 \%)$ & $13(4.1 \%)$ & \\
\hline Liver & $6(3.5 \%)$ & $4(4.0 \%)$ & $5(1.6 \%)$ & \\
\hline Prostate & $2(1.2 \%)$ & $6(5.9 \%)$ & $4(1.3 \%)$ & \\
\hline Other & $21(12.1 \%)$ & $14(13.9 \%)$ & 27 (8.6\%) & \\
\hline Missing & 39 & 24 & 36 & \\
\hline Stomach pain & & & & 0.001 \\
\hline Yes & 49 (28.3\%) & 9 (9.0\%) & $14(4.5 \%)$ & \\
\hline No & 124 (71.7\%) & 91 (91.0\%) & $300(95.5 \%)$ & \\
\hline Missing & 0 & 1 & 0 & \\
\hline Stomach ulcer & & & & 0.001 \\
\hline Yes & 77 (44.8\%) & 13 (13.0\%) & $5(1.6 \%)$ & \\
\hline No & 95 (55.2\%) & 87 (87.0\%) & 309 (98.4\%) & \\
\hline Missing & 1 & 1 & 0 & \\
\hline Physical activity levels ${ }^{2)}$ & & & & 0.001 \\
\hline Inactive & 50 (29.1\%) & $26(26.0 \%)$ & $46(14.6 \%)$ & \\
\hline Minimally active & $122(70.9 \%)$ & $73(73.0 \%)$ & 251 (79.9\%) & \\
\hline HEPA active & $0(0.0 \%)$ & $1(1.0 \%)$ & 17 (5.4\%) & \\
\hline Missing & 1 & 1 & 0 & \\
\hline
\end{tabular}

${ }^{1}$ The response base differs as some cases had either irrelevant responses or no response at all.

${ }^{2)}$ Inactive: not fitting in "minimally active [at least 600 metabolic equivalents (MET)-min/week]" or "HEPA active (more than 3,000 MET-min/week)".

family histories of gastric (9.8\%), colon and/or rectal (5.2\%), breast $(9.2 \%)$, and other $(12.1 \%)$ cancers. However, participants with PC reported family histories of lung $(5.9 \%)$, breast $(6.9 \%)$, prostate $(5.9 \%)$, and other (13.9\%) cancers. Approximately $28.3 \%$ and $44.8 \%$ of participants with GC suffered from stomach pain and stom- ach ulcers, respectively. Despite significantly different levels of physical activity between groups, participants with GC and PC and controls were all considered minimally active (Table 2).

Table 3 illustrates dietary habits of participants with GC and PC and controls. In total, $38.2 \%$ of participants 
Table 3. Dietary habits of gastric and pancreatic cancer patients

\begin{tabular}{|c|c|c|c|c|}
\hline Dietary habits variables & $\begin{array}{l}\text { Gastric cancer } \\
\quad(n=173, \%)\end{array}$ & $\begin{array}{c}\text { Pancreatic cancer } \\
(n=101, \%)\end{array}$ & $\begin{array}{c}\text { Control } \\
(n=314, \%)\end{array}$ & $P$-value \\
\hline Number of main meals & & & & 0.019 \\
\hline One meal & $10(5.8 \%)$ & $7(6.9 \%)$ & 25 (8.0\%) & \\
\hline Two meals & $66(38.2 \%)$ & $36(35.6 \%)$ & 152 (48.4\%) & \\
\hline Three meals & 95 (54.9\%) & $58(57.4 \%)$ & $129(41.1 \%)$ & \\
\hline More than three meals & $2(1.2 \%)$ & $0(0.0 \%)$ & $8(2.5 \%)$ & \\
\hline Skipped meals & & & & 0.057 \\
\hline No & $97(56.1 \%)$ & 55 (55.0\%) & 138 (44.1\%) & \\
\hline Breakfast & $22(12.7 \%)$ & $11(11.0 \%)$ & 34 (10.9\%) & \\
\hline Lunch & $5(2.9 \%)$ & $8(8.0 \%)$ & $13(4.2 \%)$ & \\
\hline Dinner & $38(22.0 \%)$ & 19 (19.0\%) & $85(27.2 \%)$ & \\
\hline Breakfast and lunch & $4(2.3 \%)$ & $2(2.0 \%)$ & $21(6.7 \%)$ & \\
\hline Breakfast and dinner & $5(2.9 \%)$ & $5(5.0 \%)$ & $19(6.1 \%)$ & \\
\hline Lunch and dinner & $2(1.2 \%)$ & $0(0.0 \%)$ & $3(1.0 \%)$ & \\
\hline Missing ${ }^{1)}$ & 0 & 1 & 1 & \\
\hline Number of snacks & & & & 0.002 \\
\hline One & 79 (45.7\%) & $42(42.0 \%)$ & 111 (35.7\%) & \\
\hline Two & $56(32.4 \%)$ & 37 (37.0\%) & $117(37.6 \%)$ & \\
\hline Three & $2(1.2 \%)$ & $2(2.0 \%)$ & 32 (10.3\%) & \\
\hline More than three & $2(1.2 \%)$ & $1(1.0 \%)$ & $5(1.6 \%)$ & \\
\hline No & $34(19.7 \%)$ & $18(18.0 \%)$ & $46(14.8 \%)$ & \\
\hline Missing & 0 & 1 & 3 & \\
\hline Number of meals eaten outside & & & & 0.001 \\
\hline No & $42(24.3 \%)$ & $25(24.8 \%)$ & 119 (37.9\%) & \\
\hline Less than once/month & $34(19.7 \%)$ & $24(23.8 \%)$ & $85(27.1 \%)$ & \\
\hline $1 \sim 3$ times/month & $35(20.2 \%)$ & $25(24.8 \%)$ & $62(19.7 \%)$ & \\
\hline 1 3 times/week & $43(24.9 \%)$ & 20 (19.8\%) & 32 (10.2\%) & \\
\hline $4 \sim 6$ times/week & $12(6.9 \%)$ & $4(4.0 \%)$ & $5(1.6 \%)$ & \\
\hline Daily & 7 (4.0\%) & $3(3.0 \%)$ & $11(3.5 \%)$ & \\
\hline Daily water amount & & & & 0.002 \\
\hline $1 \sim 3$ cups & $36(20.9 \%)$ & $23(22.8 \%)$ & $28(8.9 \%)$ & \\
\hline $3 \sim 5$ cups & $51(29.7 \%)$ & 33 (32.7\%) & $103(32.9 \%)$ & \\
\hline More than 5 cups & $83(48.3 \%)$ & $45(44.6 \%)$ & $181(57.8 \%)$ & \\
\hline I don't know & $2(1.2 \%)$ & $0(0.0 \%)$ & $1(0.3 \%)$ & \\
\hline Missing & 1 & 0 & 4 & \\
\hline Eating problem & & & & 0.001 \\
\hline No & 131 (75.7\%) & $84(83.2 \%)$ & 281 (89.5\%) & \\
\hline Yes & $42(24.3 \%)$ & $17(16.8 \%)$ & 33 (10.5\%) & \\
\hline Type of eating problem & & & & 0.001 \\
\hline Swallowing & $7(4.0 \%)$ & $3(3.0 \%)$ & $10(3.2 \%)$ & \\
\hline $\begin{array}{l}\text { Not feeling the taste and } \\
\text { smell of the food }\end{array}$ & $4(2.3 \%)$ & $0(0.0 \%)$ & $5(1.6 \%)$ & \\
\hline Loss of appetite & $3(1.7 \%)$ & $4(4.0 \%)$ & $9(2.9 \%)$ & \\
\hline Way of eating & $3(1.7 \%)$ & $1(1.0 \%)$ & $1(0.3 \%)$ & \\
\hline Add salt & $19(11.0 \%)$ & $5(5.0 \%)$ & $5(1.6 \%)$ & \\
\hline Chewing & $5(2.9 \%)$ & $4(4.0 \%)$ & $2(0.6 \%)$ & \\
\hline Vomiting & $1(0.6 \%)$ & $0(0.0 \%)$ & $1(0.3 \%)$ & \\
\hline No & $131(75.7 \%)$ & $84(83.2 \%)$ & 281 (89.5\%) & \\
\hline
\end{tabular}

${ }^{11}$ The response base differs as some cases had either irrelevant responses or no response at all.

with GC and $35.6 \%$ of participants with PC consumed two main meals/d, compared with $48.4 \%$ of controls. Over half of participants with GC and PC consumed three main meals/d, compared with only $41.1 \%$ for controls. For participants in all groups, breakfast and dinner were the most frequently skipped meals. Furthermore, the number of snacks consumed daily and the number of meals consumed outside significantly differed between participants with GC and PC and controls. In addition, participants with GC and PC consumed significantly less water daily than controls (consumption of more than 5 cups of water daily: GC cases, $48.3 \%$; PC cases, $44.6 \%$; controls, $57.8 \%$ ). The number and types of eating problems significantly differed between groups. 
Table 4. Logistic regression analysis of potential predictors of gastric and pancreatic cancer

\begin{tabular}{|c|c|c|c|c|c|c|c|c|}
\hline \multirow{3}{*}{ Potential predictors } & \multicolumn{4}{|c|}{ Gastric cancer } & \multicolumn{4}{|c|}{ Pancreatic cancer } \\
\hline & \multirow{2}{*}{$\mathrm{OR}^{1)}$} & \multicolumn{2}{|c|}{$95 \% \mathrm{CI}$} & \multirow{2}{*}{$P$-value } & \multirow{2}{*}{$\mathrm{OR}^{1)}$} & \multicolumn{2}{|c|}{$95 \% \mathrm{CI}$} & \multirow{2}{*}{$P$-value } \\
\hline & & Lower & Upper & & & Lower & Upper & \\
\hline \multicolumn{9}{|c|}{ Cancer type for patient's family } \\
\hline Gastric & 2.30 & 0.79 & 6.72 & 0.127 & 0.71 & 0.17 & 2.91 & 0.635 \\
\hline Colon and/or rectal & 0.85 & 0.27 & 2.66 & 0.774 & 0.10 & 0.01 & 0.91 & 0.041 \\
\hline Pancreatic & 1.53 & 0.04 & 52.66 & 0.814 & 6.86 & 0.23 & 206.04 & 0.267 \\
\hline Bone cancer & 1.66 & 0.33 & 8.44 & 0.538 & 1.51 & 0.26 & 8.95 & 0.648 \\
\hline Lung & 0.59 & 0.15 & 2.30 & 0.443 & 1.06 & 0.27 & 4.10 & 0.932 \\
\hline Leukaemia & 0.36 & 0.07 & 1.71 & 0.196 & 0.22 & 0.02 & 2.06 & 0.184 \\
\hline Breast & 1.44 & 0.49 & 4.23 & 0.506 & 1.08 & 0.31 & 3.75 & 0.909 \\
\hline Liver & 1.01 & 0.23 & 4.43 & 0.991 & 0.96 & 0.19 & 4.81 & 0.962 \\
\hline Prostate & 0.34 & 0.05 & 2.26 & 0.262 & 2.18 & 0.46 & 10.27 & 0.324 \\
\hline Other & \multicolumn{4}{|c|}{1 Referent } & \multicolumn{4}{|c|}{1 Referent } \\
\hline \multicolumn{9}{|l|}{ Number of main meals } \\
\hline One meal & 0.29 & 0.04 & 2.29 & 0.240 & - & - & - & - \\
\hline Two meals & 0.35 & 0.05 & 2.33 & 0.279 & - & - & - & - \\
\hline Three meals & 0.80 & 0.13 & 5.16 & 0.816 & - & - & - & - \\
\hline More than three meals & \multicolumn{4}{|c|}{1 Referent } & \multicolumn{4}{|c|}{1 Referent } \\
\hline \multicolumn{9}{|l|}{ Number of snacks } \\
\hline One & 0.62 & 0.33 & 1.14 & 0.125 & 0.71 & 0.34 & 1.49 & 0.364 \\
\hline Two & 0.44 & 0.23 & 0.85 & 0.015 & 0.49 & 0.23 & 1.08 & 0.076 \\
\hline Three & 0.04 & 0.01 & 0.23 & 0.001 & 0.08 & 0.02 & 0.40 & 0.002 \\
\hline More than three & 0.16 & 0.02 & 1.11 & 0.064 & 0.18 & 0.02 & 2.14 & 0.175 \\
\hline No & \multicolumn{4}{|c|}{1 Referent } & \multicolumn{4}{|c|}{1 Referent } \\
\hline \multicolumn{9}{|c|}{ Number of meals eaten outside } \\
\hline No & 0.31 & 0.09 & 0.99 & 0.048 & 0.4 & 0.09 & 1.89 & 0.249 \\
\hline Less than once/month & 0.39 & 0.12 & 1.29 & 0.123 & 0.81 & 0.17 & 3.83 & 0.792 \\
\hline $1 \sim 3$ times/month & 0.53 & 0.16 & 1.71 & 0.286 & 1.07 & 0.23 & 5.02 & 0.932 \\
\hline $1 \sim 3$ times/week & 1.95 & 0.59 & 6.47 & 0.273 & 2.51 & 0.52 & 12.11 & 0.252 \\
\hline $4 \sim 6$ times/week & 3.40 & 0.74 & 15.70 & 0.117 & 3.24 & 0.44 & 23.62 & 0.247 \\
\hline Daily & \multicolumn{4}{|c|}{1 Referent } & \multicolumn{4}{|c|}{1 Referent } \\
\hline
\end{tabular}

${ }^{1)}$ Adjusted for: age, gender, smoking status, family history of cancer, health problem, type of health problem and physical activity.

Table 4 shows that the odd ratios for consumption of two snacks (OR=0.44, 95\% CI: 0.23 0.85), three snacks (OR $=0.04,95 \%$ CI: $0.01 \sim 0.23$ ), and no meals outside $(\mathrm{OR}=0.31,95 \% \mathrm{CI}: 0.09 \sim 0.99)$ were inversely associated with GC risk. Furthermore, the risk of PC were inversely associated with the odds of a family history of colon and/or rectal cancer (OR $=0.10,95 \%$ CI: $0.01 \sim 0.91)$ and consumption of three snacks $(\mathrm{OR}=0.08,95 \% \mathrm{CI}$ : $0.02 \sim 0.40$ ).

\section{DISCUSSION}

This study aimed to assess the main demographic and lifestyle factors of participants with GC and PC. Furthermore, it aimed to investigate the association between these lifestyle factors and dietary habits and the risk of GC and PC among Jordanians.

Many risk factors associated with GC and PC. Being overweight or obese increases the risk of many cancers, therefore obtaining and maintaining a healthy weight may lower the risk. Our study showed that both weight at diagnosis and BMI were reduced in participants with GC and PC compared with pre-diagnosis. Most participants with GC $(57.1 \%)$ and PC $(52.6 \%)$ had a BMI range of 18.5 24.9, which is considered normal. This could be attributed to weight loss resulting from the diseases. A similar result was reported by Okada et al. (2017) who found that $69.1 \%$ of GC patients had a normal BMI in range of 18.5 24.9 (Okada et al., 2017). However, Bosetti et al. (2013) found that $39.1 \%$ of participants with PC had a BMI of 20 25.

Smoking is the most important risk factor for GC and PC. Both the number of cigarettes smoked per day and the duration of smoking in years were higher for participants with GC and PC compared with controls. The total of participants who smoked (current, previous, and passive smokers) was $52 \%$ for participants with GC and $46.5 \%$ for participants with PC. Similarly, previous studies have shown that a high percentage $(66.3 \%)$ of patients with GC is smokers (Okada et al., 2017) and that $48 \%$ of PC patients are current or previous smokers 
(Keane et al., 2014). Further studies have reported that PC is not affected by smoking (OR $=0.60,95 \% \mathrm{CI}$ : 0.30 $\sim 1.19, P=0.141$ ) (Jo et al., 2015), and that active and passive smoking may play an important role in the development of cardial stomach cancer (Mao et al., 2002). However, Duan et al. (2009) did not report any evidence that passive smoking had any appreciable effect on oesophageal or gastric adenocarcinomas.

Regular physical activity may lower cancer risk. Several studies have demonstrated that avoiding a sedentary lifestyle and participating in physical activities is helpful for reducing the risk of digestive system cancers (Tayyem et al., 2013; Tajabadi et al., 2019). In the current study, most participants with GC and PC (70.9\% and $73.0 \%$, respectively) were considered minimally active (physical activity level between $600 \sim 3,000$ MET-min/week) and were significantly less active than the control group. Similarly, Zhang et al. (2009) showed that participants with PC were less physically active than controls. Tajabadi et al. (2019) stated that the type and intensity of physical activity associated with a protective effect against gastrointestinal cancer is unknown. Furthermore, the exact underlying mechanisms linking physical activity to digestive system cancers are unknown. However, different mechanisms have been suggested. Physical activity may reduce growth factor 1 levels, which is associated with carcinogenesis, reduce leptin and increases adiponectin levels in serum, regulate hormones in blood circulation, increase sex hormone binding protein levels, promote anti-oxidant defence, and modulate immune system function. Furthermore, water intake due to physical activity may be associated with digestive system cancer risk. Water plays a role in softening content in the gut, thus increasing waste particle transit time and diluting carcinogens that can decrease the risk of colorectal cancer (Tajabadi et al., 2019).

Approximately $55.4 \%$ of participants with PC suffered from other health problems, the most common of which was diabetes mellitus (35.6\%). Previously, Zhang et al. (2009) found that $27 \%$ of patients with PC suffered from diabetes mellitus, whereas Bosetti et al. (2013) showed that $18.5 \%$ of patients with PC had diabetes mellitus. Our study revealed that $9.9 \%$ of participants with PC suffered from heart problems. However, researchers in the field of PC have stated that use of beta-blockers used in heart failure may suppress cancer invasion and proliferation (Zhang et al., 2010).

Moreover, $48.6 \%$ of participants with GC and $48.5 \%$ of participants with PC had a family history of cancer. Previous studies have shown a family history of cancer increases risk of GC (Gajalakshmi and Shanta, 1996) and PC (McGuigan et al., 2018). However, a family history of colon and/or rectal cancer was inversely associated with PC $(\mathrm{OR}=0.10,95 \% \mathrm{CI}: 0.01 \sim 0.91)$. This may be attributed to the lower number of colon and/or rectal cancers recorded in our study. Consistent with our findings, Silverman (2001) found that a family history of cancer is associated with a $30 \%$ increase in risk of PC. Silverman (2001) showed that subjects with a family history of PC $(\mathrm{OR}=3.2)$ and colon cancer $(\mathrm{OR}=1.7)$ had a significantly higher risk of PC. In the current study, stomach pain and ulcers were significantly higher in both participants with GC and PC than controls. Several previous studies have shown that stomach pain and ulcers are significantly higher in patients with PC (Bosetti et al., 2013; Keane et al., 2014).

Most participants with cancer consumed three main meals and one snack per day. However, the American Cancer Society recommends that cancer patients instead eat several small snacks throughout the day (Rock et al., 2012). We found that consuming two snacks $(\mathrm{OR}=0.44$, 95\% CI: $0.23 \sim 0.85)$ and three snacks (OR=0.04, 95\% CI: $0.01 \sim 0.23)$ decreased the risk of GC. Furthermore, consuming three snacks ( $\mathrm{OR}=0.08,95 \% \mathrm{CI}: 0.02 \sim 0.40$ ) was associated with a lower risk of PC. Dinner was the most skipped meal for participants with GC and PC. In contrast to this result, Lim et al. (2012) reported that breakfast was the most skipped meals for patients with GC in Korea. Furthermore, in the current study, the numbers of meals eaten outside by participants with GC and PC were significantly higher than for controls. As expected, we found that not eating meals outside $(\mathrm{OR}=$ 0.31, 95\% CI: $0.09 \sim 0.99$ ) decreased the risk of GC. In addition, excessive consumption of salt can increase risk of GC (Okada et al., 2017). In this study, adding salt to food was the most predominant eating problem reported by participants with GC.

Several studies have shown that water intake may play a role in reducing the risk of some digestive cancers. Water helps soften gut content, to increase waste particle transit time and dilute carcinogens, both of which decrease the risk of colorectal cancer (Tayyem et al., 2013; Tajabadi et al., 2019). In this study, fewer participants with cancer consumed more than 5 cups of water per day than controls.

The strengths of this study include the populationbased design, relatively large sample size, and separate investigation of GC and PC cases. Furthermore, this is the first study to evaluate GC and PC risk factors using a case-control study among Jordanians. Our study also had several limitations. For example, data may be subject to recall bias, and several factors associated with GC and PC were not measured (e.g. H. pylori infection).

In conclusion, the data of this study suggest that different dietary and lifestyle factors affect risk of GC and PC. Indeed, there was an association between certain lifestyle factors, dietary habits and risk of GC and PC among Jordanians. Our findings offer insight for further prospective investigations and for creating effective strategy to pre- 
vent GC and PC.

\section{ACKNOWLEDGEMENTS}

The authors would like to thank The Hashemite University for funding the research projects. We would like to express our thanks and gratitude for Eng Israa Al-Natsheh for help in data collection.

\section{AUTHOR DISCLOSURE STATEMENT}

The authors declare no conflict of interest.

\section{AUTHORSHIP}

RT and TA were responsible for the study conception and design and responsible for development of the methodology. SA, TA, AH, and YA were responsible for the acquisition of data. NA, RT, and SA were responsible for analysis and interpretation of data. NA, RT, KB, and SA were responsible for drafting the manuscript. All the authors were responsible for reviewing and/or revising the manuscript.

\section{REFERENCES}

2018 Physical Activity Guidelines Advisory Committee. 2018 Physical Activity Guidelines Advisory Committee Scientific Report. 2018 [cited 2020 Oct 22]. Available from: https:// health.gov/sites/default/files/2019-09/PAG_Advisory_Com mittee_Report.pdf

American Cancer Society. Cancer facts \& figures 2020. 2020 [cited 2020 Oct 20]. Available from: https://www.cancer.org/ content/dam/cancer-org/research/cancer-facts-and-statistic s/annual-cancer-facts-and-figures/2020/cancer-facts-and-fig ures-2020.pdf

Bosetti C, Lucenteforte E, Bracci PM, Negri E, Neale RE, Risch $\mathrm{HA}$, et al. Ulcer, gastric surgery and pancreatic cancer risk: an analysis from the International Pancreatic Cancer Case-Control Consortium (PanC4). Ann Oncol. 2013. 24:2903-2910.

Carcas LP. Gastric cancer review. J Carcinog. 2014. 13:14. https:// doi.org/10.4103/1477-3163.146506

Cormie P, Zopf EM, Zhang X, Schmitz KH. The impact of exercise on cancer mortality, recurrence, and treatment-related adverse effects. Epidemiol Rev. 2017. 39:71-92.

Costello E. A metabolomics-based biomarker signature discriminates pancreatic cancer from chronic pancreatitis. Gut. 2018. 67:2-3.

Duan L, Wu AH, Sullivan-Halley J, Bernstein L. Passive smoking and risk of oesophageal and gastric adenocarcinomas. Br J Cancer. 2009. 100:1483-1485.

Ferlay J, Soerjomataram I, Dikshit R, Eser S, Mathers C, Rebelo $\mathrm{M}$, et al. Cancer incidence and mortality worldwide: sources, methods and major patterns in GLOBOCAN 2012. Int J Cancer. 2015. 136:E359-E386.

Ferronha I, Castro C, Carreira H, Bento MJ, Carvalho I, Peleteiro
B, et al. Prediagnosis lifestyle exposures and survival of gastric cancer patients: a cohort study from Portugal. Br J Cancer. 2012. 107:537-543.

Forde C. Exercise prescription for the prevention and treatment of disease. 2018 [cited 2020 Sep 25]. Available from: https:// ugc.futurelearn.com/uploads/files/bc/c5/bcc53b14-ecle-4d 90-88e3-1568682f32ae/IPAQ PDF.pdf

Gajalakshmi CK, Shanta V. Lifestyle and risk of stomach cancer: a hospital-based case-control study. Int J Epidemiol. 1996. 25:1146-1153.

Islami F, Goding Sauer A, Miller KD, Siegel RL, Fedewa SA, Jacobs EJ, et al. Proportion and number of cancer cases and deaths attributable to potentially modifiable risk factors in the United States. CA Cancer J Clin. 2018. 68:31-54.

Jo SR, Joh JY, Jeong JR, Kim S, Kim YP. Health behaviors of Korean gastric cancer survivors with hypertension: a propensity analysis of KNHANES III-V (2005-2012). PLoS One. 2015. 10:e0126927. https://doi.org/10.1371/journal.pone.0126927

Keane MG, Horsfall L, Rait G, Pereira SP. A case-control study comparing the incidence of early symptoms in pancreatic and biliary tract cancer. BMJ Open. 2014. 4:e005720. http://doi. org/10.1136/bmjopen-2014-005720

Lee R, Nieman D. Nutritional assessment. 6th ed. McGraw-Hill Companies, Inc., New York, NY, USA. 2013. p 176.

Lee YY, Derakhshan MH. Environmental and lifestyle risk factors of gastric cancer. Arch Iran Med. 2013. 16:358-365.

Lim H, Cho G, Kim S. Evaluation of nutrient intake and diet quality of gastric cancer patients in Korea. Nutr Res Pract. 2012. 6:213-220

Mao Y, Hu J, Semenciw R, White K; Canadian Cancer Registries Epidemiology Research Group. Active and passive smoking and the risk of stomach cancer, by subsite, in Canada. Eur J Cancer Prev. 2002. 11:27-38.

McGuigan A, Kelly P, Turkington RC, Jones C, Coleman HG, McCain RS. Pancreatic cancer: a review of clinical diagnosis, epidemiology, treatment and outcomes. World J Gastroenterol. 2018. 24:4846-4861.

Okada E, Ukawa S, Nakamura K, Hirata M, Nagai A, Matsuda K, et al. Demographic and lifestyle factors and survival among patients with esophageal and gastric cancer: The Biobank Japan Project. J Epidemiol. 2017. 27:S29-S35.

Rock CL, Doyle C, Demark-Wahnefried W, Meyerhardt J, Courneya KS, Schwartz AL, et al. Nutrition and physical activity guidelines for cancer survivors. CA Cancer J Clin. 2012. 62:243-274.

Sallis JF, Haskell WL, Wood PD, Fortmann SP, Rogers T, Blair SN, et al. Physical activity assessment methodology in the FiveCity Project. Am J Epidemiol. 1985. 121:91-106.

Silverman DT. Risk factors for pancreatic cancer: a case-control study based on direct interviews. Teratog Carcinog Mutagen. 2001. 21:7-25.

Sundelöf M, Lagergren J, Ye W. Patient demographics and lifestyle factors influencing long-term survival of oesophageal cancer and gastric cardia cancer in a nationwide study in Sweden. Eur J Cancer. 2008. 44:1566-1571.

Tajabadi Z, Akbari ME, Hafez AA. Physical activity and gastrointestinal cancer risk: a review. Acta Medica Bulgarica. 2019. 46:57-67.

Tayyem RF, Shehadeh IN, Abumweis SS, Bawadi HA, Hammad SS, Bani-Hani KE, et al. Physical inactivity, water intake and constipation as risk factors for colorectal cancer among adults in Jordan. Asian Pac J Cancer Prev. 2013. 14:5207-5212.

Thompson JK, Jarvie GJ, Lahey BB, Cureton KJ. Exercise and obesity: etiology, physiology, and intervention. Psychol Bull. 1982. 91:55-79.

Thornton A, Lee P, Fry J. Differences between smokers, ex-smokers, passive smokers and non-smokers. J Clin Epidemiol. 1994. 
47:1143-1162.

Trivers KF, De Roos AJ, Gammon MD, Vaughan TL, Risch HA, Olshan AF, et al. Demographic and lifestyle predictors of survival in patients with esophageal or gastric cancers. Clin Gastroenterol Hepatol. 2005. 3:225-230.

WHO. Diet, nutrition and the prevention of chronic diseases. WHO Technical Report Series 916, World Health Organization, Geneva, Switzerland. 2002 [cited 2020 Sep 20]. Available from: https://apps.who.int/iris/bitstream/handle/10665/ 42665/WHO_TRS_916.pdf;jsessionid =6DDAB9216C4F 2659AB15335C56E777E7? sequence $=1$

World Cancer Research Fund/American Institute for Cancer Research. Diet, nutrition, physical activity and cancer: a global perspective. 2018 [cited 2020 Oct 20]. Available from: https://
www.wcrf.org/sites/default/files/Summary-of- Third-ExpertReport-2018.pdf

Zhang D, Ma QY, Hu HT, Zhang M. $\beta 2$-adrenergic antagonists suppress pancreatic cancer cell invasion by inhibiting CREB, NFKB and AP-1. Cancer Biol Ther. 2010. 10:19-29.

Zhang J, Dhakal IB, Gross MD, Lang NP, Kadlubar FF, Harnack LJ, et al. Physical activity, diet, and pancreatic cancer: a population-based, case-control study in Minnesota. Nutr Cancer. 2009. 61:457-465.

Zhao LL, Huang H, Wang Y, Wang TB, Zhou H, Ma FH, et al. Lifestyle factors and long-term survival of gastric cancer patients: a large bidirectional cohort study from China. World J Gastroenterol. 2020. 26:1613-1627. 